\title{
Characteristics analysis of silicon carbide based 1-D Photonic crystal with 2 layered unit cell using MATLAB
}

\author{
Avik Chakraborty \\ Assistant Professor, Electronics \& Communication Engg. Dept., Bengal Institute of Technology \& Management, \\ Bolpur, Birbhum. Contact Address: Senpara, Kalitala Road, PO+Dist- Jalpaiguri, Pin-735101, West Bengal,
}

\begin{abstract}
Silicon carbide based 1-D Photonic crystals with 2 layered unit cell are analyzed in this paper. The transmission characteristics of photonic crystals are computed by transfer matrix method and performance of such photonic crystals of different material combinations are also compared, and after comparison some one able to know the characteristics such as pass band width, stop band width, centre band gap of a light wave of a particular wave length traveling through this 2 layered unit cell photonic crystal in nano meter range where one layer of this unit cell must be made of semiconductor material named silicon carbide ( $\mathrm{SiC}$ ).

Key word: 1-D photonic crystal, transmission coefficient, light wave.
\end{abstract}

\section{Introduction}

In this paper first to know about photonic crystal [1] the statement is like that periodic optical nanostructures that are designed to affect the motion of photons in a similar way that periodicity of a semiconductor crystal affects the motion of electrons. such photonic crystals with photonic band gap (for some frequency range, a photonic crystal prohibits the propagation of electromagnetic waves of any polarization travelling in any direction from any source, then we say that the crystal has a complete photonic band gap) preventing light from propagating in certain directions with specified frequencies In order to construct these crystals in the optical regime, suitable nanofabrication techniques have to be developed and demonstrated, including high resolution electron beam lithography and anisotropic chemically assisted ion beam etching. These periodic dielectric structures are expected to exhibit interesting properties in both fields of physics and engineering. Silicon Carbide ( $\mathrm{SiC}$ ) being an important semiconductor in the situation. The basic characteristics of Silicon Carbide ( $\mathrm{SiC})$ - based photonic crystals will be analyzed .

\section{One Dimensional Photonic Crystal (Phe)}

In a 1-D Photonic Crystal layers of different dielectric constant may be deposited or adhered together to form a band gap in a single direction.A Bragg grating is an example of this type of photonic crystal .This type of photonic crystal systems exhibits three important phenomena i) Photonic Band gap, ii)Localized mode iii) surface state ,because the index contrast is only along one direction, the band gaps and bound states are limited to that direction .By the help of this concept this characteristics analysis is being processed.

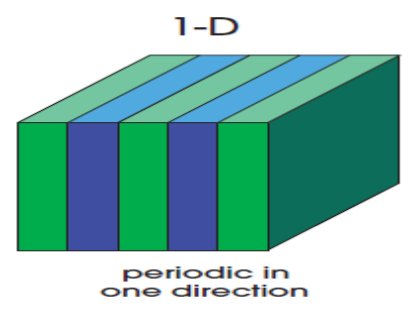

Fig (1)-1-D Photonic Crystal

\section{Reason behind the choosing of Silicon Carbide ( $\mathrm{SiC}$ ) as a semiconductor material}

$\mathrm{SiC}$ (wide forbidden energy gap of $2.86 \mathrm{eV} @ 302 \mathrm{~K}$ ) has been emerged as an attractive substrate for high power devices due to its larger band gap, higher break down strength, high thermal conductivity and high electron saturation drift velocity. Most important advantage of $\mathrm{SiC}$ over other wide band gap semiconductor is that $\mathrm{SiC}$ can be thermally oxidized to form an insulator Silicon die oxide. Although the refractive index of $\mathrm{SiC}$ is lower than that of a conventional semiconductor such as $\mathrm{GaAs}$ or $\mathrm{Si}$, a wide photonic band gap , a broadband wave guide and a high quality nanocavity comparable to those of previous photonic crystals can be obtained in Silicon Carbide based photonic crystals, for above many reason in this analysis process we choose SiC.

\section{Description}


In this paper unit cell photonic crystal has been considered which has 2 layers. The refractive indices $\left(\mathrm{n}_{1}, \mathrm{n}_{2}\right)$ of the two materials are related to the thickness of the layers $\left(d_{1}, d_{2}\right)$ as

$$
\mathrm{n}_{1} \mathrm{~d}_{1}=\mathrm{n}_{2} \mathrm{~d}_{2}=\lambda_{0} / 4
$$

Where $\lambda_{0}=$ Free space (vacuum) wavelength, whose value is taken here as 1550 nanometer, since it is the most important wavelength for optical communication.

When an electromagnetic wave with S-polarization incidents on a unit cell of two materials a and b, the reflection and transmission coefficient can be expressed as [2]

$$
\frac{A_{R}}{A_{I}}=\frac{\sqrt{\epsilon_{a} / \mu_{a}} \cos \alpha-\sqrt{\epsilon_{b} / \mu_{b}} \cos \beta}{\sqrt{\epsilon_{a} / \mu_{a}} \cos \alpha+\sqrt{\epsilon_{b} / \mu_{b}} \cos \beta}
$$

$$
\frac{A_{T}}{A_{I}}=\frac{2 \sqrt{\epsilon_{a} / \mu_{a}} \cos \alpha}{\sqrt{\epsilon_{a} / \mu_{a}} \cos \alpha+\sqrt{\epsilon_{b} / \mu_{b}} \cos \beta}
$$
the interface.

Where $\mathrm{A}$ is the complex amplitude of the electric field, $\alpha$ and $\beta$ are angle of incidence and refraction at Here S- polarized wave has its electric field vector confined perpendicular to the plane of incidence. Here electric field $\mathrm{E}$ is in the $\mathrm{y}$ direction, so $\tilde{\mathrm{E}}=(0, \mathrm{~A}, 0)$. Using $\mathrm{S}$-polarization [equation (2) and (3)] we get the equation (4)

$$
\begin{aligned}
C_{m-1} e^{i k_{m} z_{m} \cos x_{m-1}} & \\
& =\frac{\sqrt{\epsilon_{m-1} / \mu_{m-1}} \cos \propto_{m-1}-\sqrt{\epsilon_{m} / \mu_{m}} \cos \propto_{m}}{\sqrt{\epsilon_{m-1} / \mu_{m-1}} \cos \propto_{m-1}+\sqrt{\epsilon_{m} / \mu_{m}} \cos \propto_{m}} A_{m-1} \\
& \times e^{-i k_{m} z_{m} \cos \alpha_{m-1}}+\frac{2 \sqrt{\epsilon_{m} / \mu_{m}} \cos \propto_{m}}{\sqrt{\epsilon_{m-1} / \mu_{m-1}} \cos \propto_{m-1}+\sqrt{\epsilon_{m} / \mu_{m}} \cos \propto_{m}} C_{m} \\
& \times e^{i k_{m} z_{m} \cos \alpha_{m}}
\end{aligned}
$$

$$
\begin{aligned}
& A_{m} e^{-i k_{m} z_{m} \cos \alpha_{m}} \\
& =\frac{2 \sqrt{\epsilon_{m-1} / \mu_{m-1}} \cos \alpha_{m-1}}{\sqrt{\epsilon_{m-1} / \mu_{m-1}} \cos \alpha_{m-1}+\sqrt{\epsilon_{m} / \mu_{m}} \cos \alpha_{m}} A_{m-1} \\
& X e^{-i k_{m} z_{m} \cos \alpha_{m-1}}+\frac{\sqrt{\epsilon_{m} / \mu_{m}} \cos \alpha_{m}-\sqrt{\epsilon_{m-1} / \mu_{m-1}} \cos \alpha_{m-1}}{\sqrt{\epsilon_{m-1} / \mu_{m-1}} \cos \alpha_{m-1}+\sqrt{\epsilon_{m} / \mu_{m}} \cos \alpha_{m}} C_{m} \\
& \mathrm{X} e^{i k_{m} z_{m} \cos \alpha_{m}}
\end{aligned}
$$

\section{And}

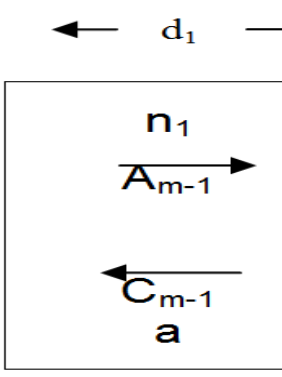

$\mathrm{m}-1$ $\mathrm{d}_{2}$

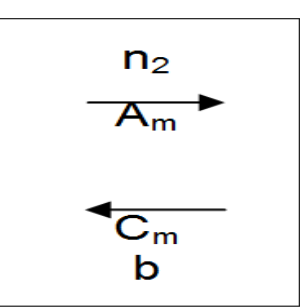

$\mathrm{m}$

Fig-(2)- Standard 2 layered unit cell Photonic Crystal

$a$ and $b$ indicates the two layers with refractive indices $n_{1}$ and $n_{2}$. 
From the figure 2, First layer is for denser material and other one is for rarer material. $d_{1}$ and $d_{2}$ are the thickness of each layer. $C_{m}$ is the amplitude in the $m^{\text {th }}$ layer for waves and $C_{m-1}$ is for $m-1$ layer that is first layer.

$A_{m}$ is the amplitude in the $\mathrm{m}^{\text {th }}$ layer for waves and $A_{m-1}$ is the amplitude in the $m-1$ layer. From equation (4) \& (5) it is possible to construct transfer matrix ,here cosine value is 0 for all cases (consider) and $\mu=1$ in all cases but $€$ value is changed for different material. Here m-1 layer signify the first layer and $\mathrm{m}$ is for second layer of an unit cell photonic crystal. By help of equation (4) and (5) first have to construct a standard unit cell whose two layers are made with $\mathrm{Si}$ and $\mathrm{SiO} 2$, after that just have to change the material from standard one and place $\mathrm{SiC}$ on that particular position and analyze the characteristics for different material combination where $\mathrm{SiC}$ is a common one.

$$
\begin{gathered}
\text { The matrix form is like that } \\
\mathrm{M}= \\
{\left[\begin{array}{l}
\mathrm{a} 1 * \exp (-\mathrm{i} * \mathrm{~km} * \mathrm{zm}) \mathrm{a} 2 * \exp (\mathrm{i} * \mathrm{~km} * \mathrm{zm}) \\
\mathrm{a} 2 * \exp (-\mathrm{i} * \mathrm{~km} * \mathrm{zm}) \mathrm{a} 1 * \exp (\mathrm{i} * \mathrm{~km} * \mathrm{zm})
\end{array}\right]\left[\begin{array}{l}
\mathrm{b} 1 * \exp (-\mathrm{i} * \mathrm{~km} 1 * \mathrm{zm} 1) \mathrm{b} 2 * \exp (\mathrm{i} * \mathrm{~km} 1 * \mathrm{zm} 1) \\
\mathrm{b} 2 * \exp (-\mathrm{i} * \mathrm{~km} 1 * \mathrm{zm} 1) \mathrm{b} 1 * \exp (\mathrm{i} * \mathrm{~km} 1 * \mathrm{zm} 1)
\end{array}\right]}
\end{gathered}
$$

Here $\mathrm{zm}=\mathrm{d}_{2}$ and $\mathrm{zm} 1=\mathrm{d}_{1}$, the general form of equation for Transfer matrix M shown in below through which transmission coefficient can be achieved.

$$
M=\left(\begin{array}{cc}
1 / t & r_{1}^{*} / t^{*} \\
r_{1} / t & 1 / t^{*}
\end{array}\right)
$$

If we apply the particular transmission coefficient for 10 numbers of unit cells then transmission coefficient of entire photonic crystal can be achieved.I

To apply 10 numbers of unit cells the standard matrix form of equation is stated below

$$
M^{N}=\left(\begin{array}{cc}
1 / t_{N} & r_{N}^{*} / t_{N}^{*} \\
r_{N} / t_{N} & 1 / t_{N}^{*}
\end{array}\right)
$$

Computation of result using different parameters

Using equation (6) transmission coefficient has been computed using matlab. For computation this paper takes the following parameters.

Free space wavelength $\left(\lambda_{0}\right)=1550$ nanometer $(\mathrm{nm})$

Number of unit cell $(\mathrm{N})=10$

$\mathrm{d}_{1}=$ Thickness of the first layer

$\mathrm{d}_{2}=$ Thickness of the second layer

$\mathrm{n}_{1}=$ Refractive index of the first layer

$\mathrm{n}_{2}=$ Refractive index of the second layer

\section{Silicon_Silica:}

\section{Discussion of results}

In case of Silicon and Silica the value of $n_{1}$ for Silicon is 3.5 and the value of $n_{2}$ for Silica is 1.5 , so by using equation (1) thickness of the first layer $\left(d_{1}\right)=1550 / 4 * 3.5=110.71 \mathrm{~nm}$ and thickness for the second layer $\left(\mathrm{d}_{2}\right)=1550 / 4 * 1.5=258.33 \mathrm{~nm}$.

In Silicon_Silica based photonic crystal the stop band lies in between $1250 \mathrm{~nm}$ to $2100 \mathrm{~nm}$ (Approx), so the value of transmission coefficient is 0 within this range. We can find Stop band width $=850 \mathrm{~nm}$. Here centre band gap is at $1680 \mathrm{~nm}$. Pass band lies in between $550 \mathrm{~nm}$ to $1250 \mathrm{~nm}$ (Approx), so Pass band width = $1250-550=700 \mathrm{~nm}$. The choosing wavelength is $1550 \mathrm{~nm}$ but centre band gap lie is at $1680 \mathrm{~nm}$. So our wavelength shifts towards the higher frequency range with respect to corresponding wavelength $\left(\lambda_{0}\right)$. After 2120 $\mathrm{nm}$ (Approx) find another Pass band till investigate of our range $2400 \mathrm{~nm}$. 


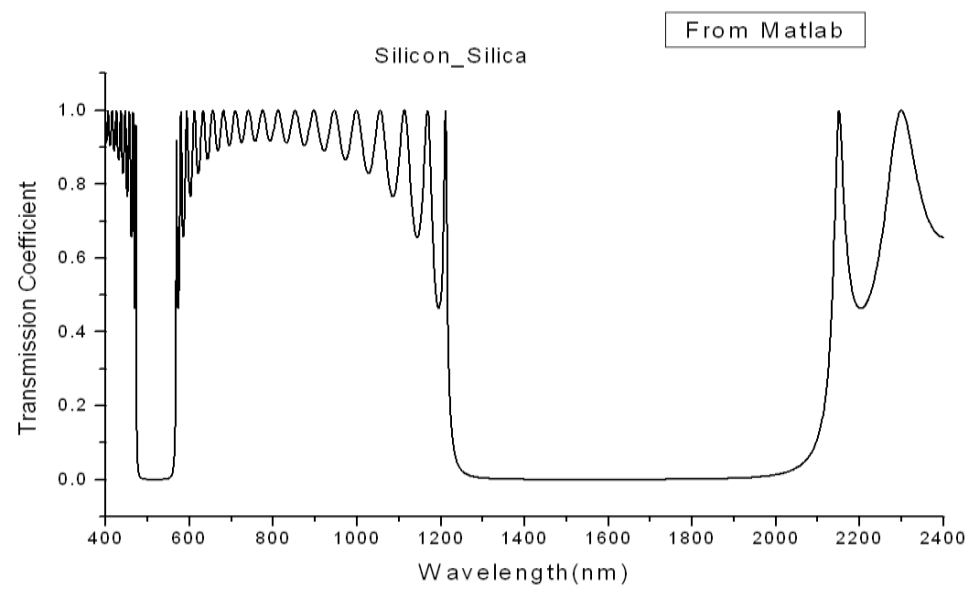

Fig3: Plot between

Wavelength(nm) Vs. Trasmission Coefficient in case of Silicon_Silica based photonic crystal containing number of unit cells $(N)=10$

\section{Silicon_Silicon Carbide:}

In the case of Silicon and Silicon Carbide the value of $n_{1}$ for Silicon is 3.5 and the value of $n_{2}$ for Silicon Carbide is 2.55 , so by using equation (1) thickness of the first layer $\left(\mathrm{d}_{1}\right)=1550 / 4 * 3.5=110.71 \mathrm{~nm}$ and thickness for the second layer $\left(\mathrm{d}_{2}\right)=1550 / 4 * 2.55=151.96 \mathrm{~nm}$.

Here transmission coefficient value is not actually 0 , its value is around 0.1 . The reason behind is that the difference between the refractive index value of this two material is so small $(3.50-2.55)=0.95$, so Stop band does not touches the 0 line. We find Pass band lies in between $550 \mathrm{~nm}$ to $1390 \mathrm{~nm}$ (Approx), so Pass band width $=840 \mathrm{~nm}$. From graph it is clearly shown that centre band gap lie is at $1580 \mathrm{~nm}$ (Approx) but our working wavelength is $1550 \mathrm{~nm}$. For this reason wavelength shifts towards the higher frequency range with respect to corresponding wavelength $\left(\lambda_{0}\right)$. Starts from $1780 \mathrm{~nm}$ (Approx) here find another Pass band till investigate of our range $2400 \mathrm{~nm}$.

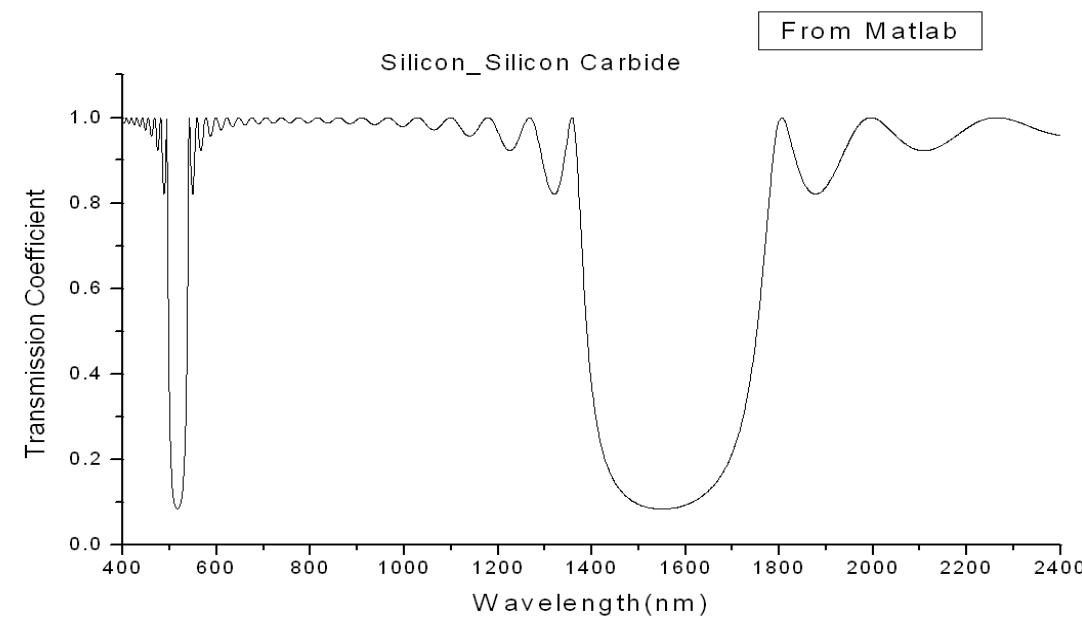

Fig4: Plot between Wavelength(nm) Vs. Transmission Coefficient in case of Silicon_Silicon Carbide based photonic crystal containing number of unit cells $(N)=10$

\section{Silicon_Silicon Carbide:}

In that case transmission coefficient value is not actually 0 , its value is around 0.1 . The reason behind is that the difference between the refractive index value of this two material is so small $(3.50-2.55)=0.95$, so Stop band does not touches the 0 line and has value (0.1). If we increase the number of unit cells from 10 to 30 , then Stop band range is within $1410 \mathrm{~nm}$ to $1730 \mathrm{~nm}$ (Approx), so Transmission Coefficient value is actually 0 and Stop band width is $320 \mathrm{~nm}$. Pass band lies in between $550 \mathrm{~nm}$ to $1410 \mathrm{~nm}$ (Approx), so Pass band width= $860 \mathrm{~nm}$. From graph it is clearly shown that centre band gap lie is at $1580 \mathrm{~nm}$ (Approx) but our working wavelength is $1550 \mathrm{~nm}$. For this reason wavelength shifts towards the higher frequency range with respect to corresponding wavelength $\left(\lambda_{0}\right)$. Starts from $1730 \mathrm{~nm}$ (Approx) here find another Pass band till investigate of our range $2400 \mathrm{~nm}$. 


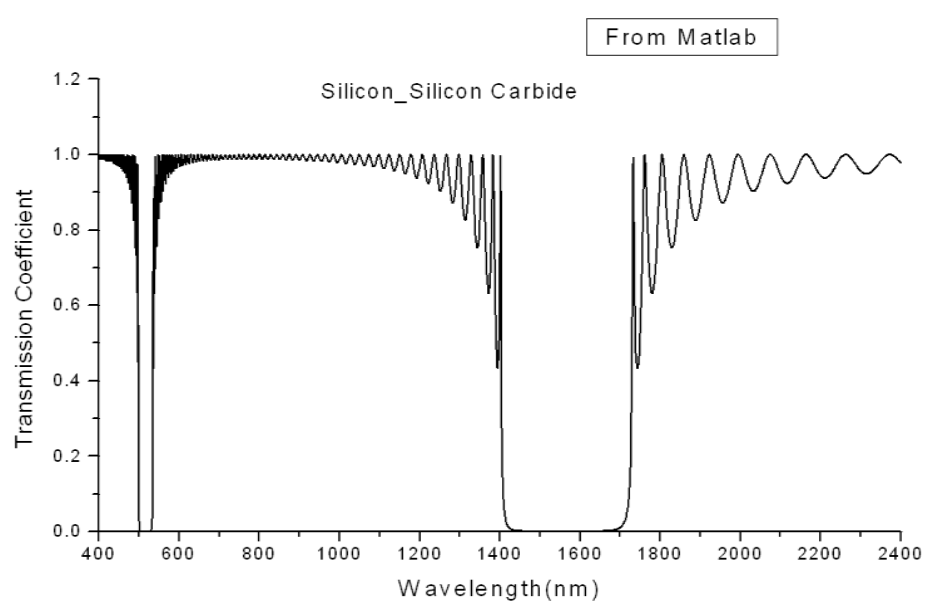

Fig5: Plot between Wavelength(nm) Vs. Transmission Coefficient in case of Silicon_Silicon Carbide based photonic crystal containing number of unit cells $(N)=30$

\section{Silicon Carbide_Air:}

Here for Silicon Carbide and Air the value of $n_{1}$ for Silicon Carbide is 2.55 and the value of $n_{2}$ for Air is 1 , so by using equation (1) thickness of the first layer $\left(\mathrm{d}_{1}\right)=1550 / 4 * 2.55=151.96 \mathrm{~nm}$ and thickness for the second layer $\left(\mathrm{d}_{2}\right)=1550 / 4 * 1=387.50 \mathrm{~nm}$.

From this plot it is clearly shown that Stop band lies in between $1230 \mathrm{~nm}$ to $2180 \mathrm{~nm}$ (Approx). Within this Stop band range transmission coefficient value is 0, here Stop band width $=2180-1230=950 \mathrm{~nm}$. Centre band gap is at $1680 \mathrm{~nm}$. Pass band lies in between $550 \mathrm{~nm}$ to $1230 \mathrm{~nm}$ (Approx). So Pass band width = $1230-$ $550=680 \mathrm{~nm}$. We work with $1550 \mathrm{~nm}$ wavelength but here find centre band gap lie is at $1680 \mathrm{~nm}$. So our wavelength shifts towards the higher frequency range with respect to corresponding wavelength $\left(\lambda_{0}\right)$. After 2180 $\mathrm{nm}$ (Approx) we find another Pass band till investigate of our range $2400 \mathrm{~nm}$.

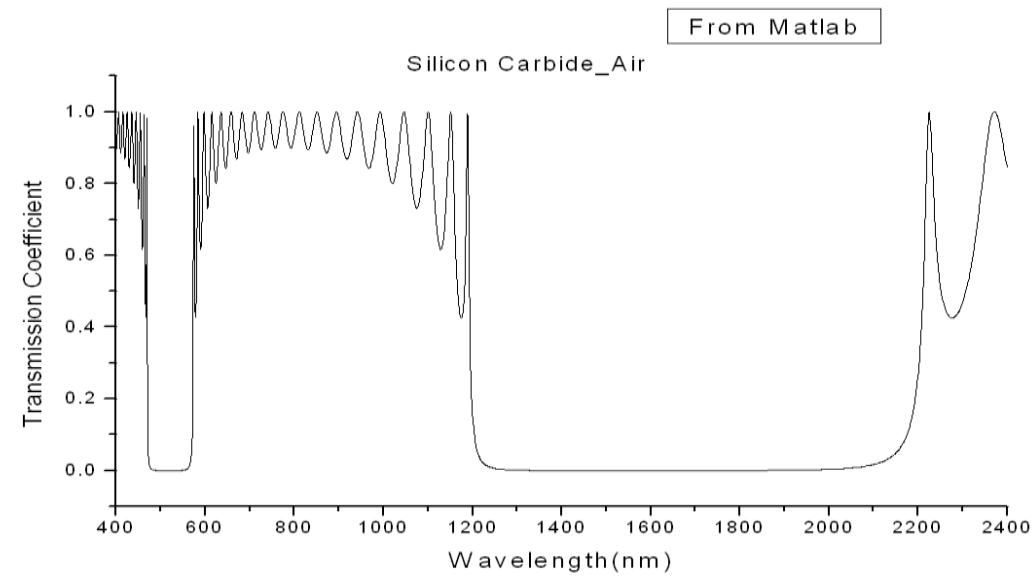

Fig6: Plot between Wavelength(nm) Vs. Transmission Coefficient in case of Silicon Carbide_Air based photonic crystal containing number of unit cells $(N)=10$

\section{Silicon Carbide_Silica:}

In that case of Silicon Carbide and Silica the value of $\mathrm{n}_{1}$ for Silicon Carbide is 2.55 and the value of $\mathrm{n}_{2}$ for Silica is 1.5 , so by using equation (1) thickness of the first layer $\left(\mathrm{d}_{1}\right)=1550 / 4 * 2.55=151.96 \mathrm{~nm}$ and thickness for the second layer $\left(\mathrm{d}_{2}\right)=1550 / 4 * 1.5=258.33 \mathrm{~nm}$.

Here the value of transmission coefficient is not actually 0 , its value is just above the 0 the reason is that the difference between the refractive index of that two material is so small $(2.55-1.50)=1.05$, that's why it is not possible to achieve the Stop band. The Pass band lies in between $550 \mathrm{~nm}$ to $1320 \mathrm{~nm}$ (Approx), so the Pass band width is $1320-550=770 \mathrm{~nm}$. In that case choosing wavelength is $1550 \mathrm{~nm}$ but the centre band gap lie is at $1600 \mathrm{~nm}$. So wavelength shifts towards the higher frequency range with respect to corresponding wavelength $\left(\lambda_{0}\right)$. After $1900 \mathrm{~nm}$ (Approx) the end range is $2400 \mathrm{~nm}$ within that region we find another Pass band lies. 


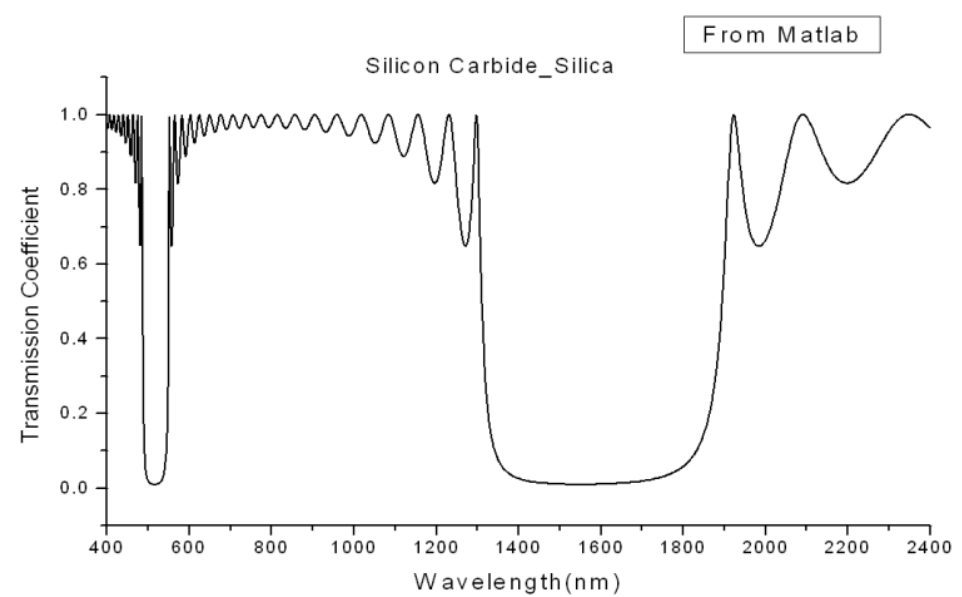

Fig7: Plot between Wavelength(nm) Vs. Transmission Coefficient in case of Silicon Carbide_Silica based photonic crystal containing number of unit cells $(N)=10$

\section{Silicon Carbide_Silica:}

Here the value of transmission coefficient is not actually 0 , its value is just above the 0 the reason is that the difference between the refractive index of that two material is so small $(2.55-1.50)=1.05$, that's why it is not possible to achieve the Stop band. To achieve the Stop band we have to increase the number of unit cells (N) from 10 to 20, then we get the actual Transmission Coefficient value 0 within the Stop band range $1320 \mathrm{~nm}$ to $1870 \mathrm{~nm}$ (Approx), so Stop band width is $1870-1320=550 \mathrm{~nm}$. Pass band lies in between $550 \mathrm{~nm}$ to $1320 \mathrm{~nm}$ (Approx), so Pass band width is $770 \mathrm{~nm}$. It is clearly shown that centre band gap lie is at $1600 \mathrm{~nm}$ (Approx) but our working wavelength is $1550 \mathrm{~nm}$. For this reason wavelength shifts towards the higher frequency range with respect to corresponding wavelength $\left(\lambda_{0}\right)$. Starts from $1870 \mathrm{~nm}$ (Approx) here find another Pass band till investigate of our range $2400 \mathrm{~nm}$.

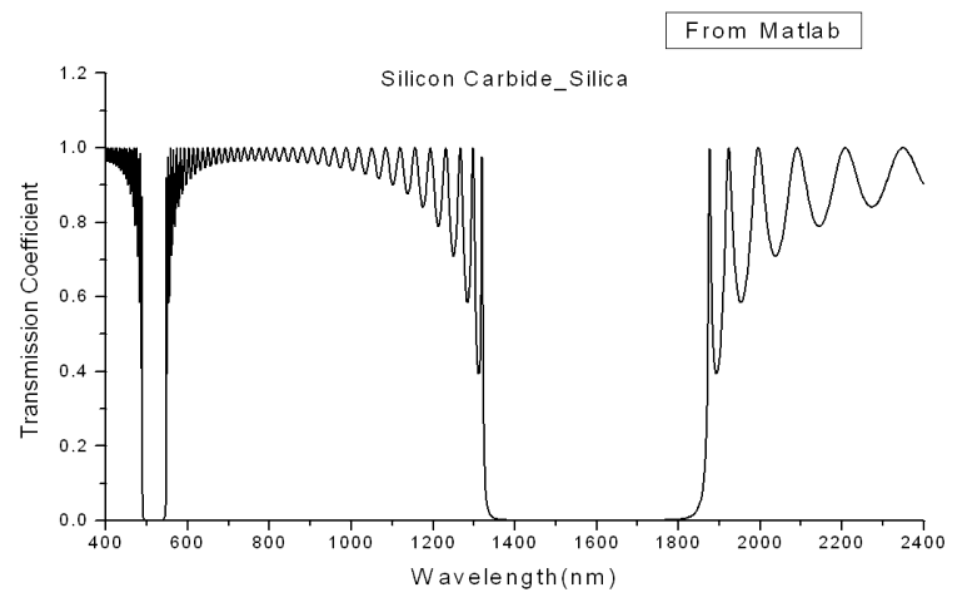

Fig8: Plot between

Wavelength(nm) Vs. Transmission Coefficient in case of Silicon Carbide_Silica based photonic crystal containing number of unit cells $(N)=20$

\section{Conclusion}

From the below, combinations of different 2 layers photonic crystal(shown in table) it is become easier to compare the various characteristics and from below table it is clearly shown that for different material combinations the characteristics of $\mathrm{SiC}$ based photonic crystal has been varying. In case of stop band there is a large variation but in pass band the variation is not so large as of stop band .In this Silicon Carbide based 1D photonic crystal with 2 layered unit cell there are particular two types of semiconductor material combinations where selectivity of that two unit cell are not so good that's why firstly impossible to get stop band, after increasing the number of unit cell the selectivity will stand in a good position and then stop band is clearly shown. 


\begin{tabular}{|c|c|c|c|c|}
\hline Material & $\begin{array}{c}\text { Stop band } \\
\text { width }(\mathrm{nm}) \\
\text { approx. }\end{array}$ & $\begin{array}{c}\text { Pass band width } \\
(\mathrm{nm}) \text { approx. }\end{array}$ & $\begin{array}{c}\text { Centre band gap } \\
(\mathrm{nm}) \text { approx. }\end{array}$ & $\begin{array}{c}\text { Deviation of } \\
\text { wavelength } \\
\text { w.r.t. } 1550 \mathrm{~nm} .\end{array}$ \\
\hline Silicon_Silica & 850 & 700 & 1680 & 130 \\
\hline Silicon_Silicon Carbide & 320 & 840 & 1580 & 30 \\
\hline Silicon Carbide_Air & 950 & 680 & 1680 & 130 \\
\hline Silicon Carbide_Silica & 550 & 770 & 1600 & 50 \\
\hline
\end{tabular}

\section{References}

[1] J. D. Joannopoulos, S. G. Johnson, J. N. Winn, R. D. Meade, "Photonic Crystals Molding the Flow of Light", Princeton University Press, 2008.

[2] J. W. Haus, "Photonic Band gap Structures" in nanometer structures: Theory, Modelling and Simulation (Ed: A. Lakhtakia), PHI, New Delhi, 2007, P. 45.

[3] "Photonic crystal", http://en.wikipedia.org/wiki/Photonic_crystal 